\title{
Comprehensive knowledge and uptake of cervical cancer screening is low among women living with HIV/AIDS in Northwest Ethiopia
}

Daniel Asfaw Erku ${ }^{1 *}$ (D) Adeladlew Kassie Netere ${ }^{1}$, Amanual Getnet Mersha², Sileshi Ayele Abebe², Abebe Basazn Mekuria ${ }^{3}$ and Sewunet Admasu Belachew ${ }^{1}$

\begin{abstract}
Background: In Ethiopia, cervical cancer is ranked as the second most common type of cancer in women and it is about 8 times more common in HIV infected women. However, data on knowledge of HIV infected women regarding cervical cancer and acceptability of screening is scarce in Ethiopia. Hence, the present study was aimed at assessing the level of knowledge of about cervical cancer and uptake of screening among HIV infected women in Gondar, northwest Ethiopia.

Methods: A cross sectional, questionnaire based survey was conducted on $302 \mathrm{HIV}$ infected women attending the outpatient clinic of University of Gondar referral and teaching hospital from March 1 to 30, 2017. Descriptive statistics, univariate and multivariate logistic regression analysis were also performed to examine factors associated with uptake of cervical cancer screening service.

Results: Overall, only 64 (21.2\%) of respondent were knowledgeable about cervical cancer and screening and only 71 (23.5\%) of respondents were ever screened in their life time. Age between 21 and 29 years old $(\mathrm{AOR}=2.78,95 \% \mathrm{Cl}=1$. 71-7.29), perceived susceptibility to develop cervical cancer ( $A O R=2.85,95 \% \mathrm{Cl}=1.89-6.16$ ) and comprehensive knowledge of cervical cancer $(A O R=3.02,95 \% \mathrm{Cl}=2.31-7.15)$ were found to be strong predictors of cervical cancer screening service uptake.

Conclusion: The knowledge and uptake of cervical cancer screening among HIV infected women was found to be very poor. Taking into consideration the heightened importance of comprehensive knowledge in boosting up the number of participants towards cervical cancer screening services, different stakeholders working on cancer and HIV/ AIDS should provide a customized health promotion intervention and awareness creation to HIV-infected women, along with improving accessibility of cervical cancer screening services in rural areas.
\end{abstract}

Keywords: Cervical cancer, Ethiopia, HIV/AIDS, Screening, Knowledge, Women

\footnotetext{
*Correspondence: daniel.asfaw05@gmail.com

'Department of Clinical Pharmacy, School of Pharmacy, College of Medicine and Health Sciences, University of Gondar, Chechela Street, Lideta Sub city Kebele 16, P.O. Box: 196, Gondar, Ethiopia

Full list of author information is available at the end of the article
} 


\section{Background}

Cancer of the cervix, mainly attributed to persistent infection with a high risk oncogenic Human papillomavirus (HRHPV), is one of the most common type of women's cancer globally, with more than $90 \%$ of new cases occurring in developing and resource-limited countries [1-3]. It is also associated with a higher rate of mortality with over 150,000 global mortality reported only in 2012, of which $87 \%$ of death occurred in developing countries $[1,2]$. In Ethiopia, cervical cancer is ranked as the second most common type of cancer in women with crude incidence rate of 16.3 per 100,000 populations annually. In 2012 only, more than 27 million women of reproductive age in Ethiopia were at risk of developing cervical cancer and jeopardized the lives of more than 4500 women [4].

Evidences showed that, immunosuppression and low CD4 counts caused by HIV infection predisposes women living with HIV infection at an increased risk for cervical cancer and the development of squamous intraepithelial lesions [5-9]. Cervical cancer is about 8 times more common in HIV infected women than none infected ones [5]. From around 35 new cases diagnosed annually per 100, 000 population in sub-Saharan African countries, about $60 \%$ of the cases diagnosed among patients living with HIV infection $[10,11]$. In a study done in southern Ethiopia, around $22 \%$ women infected with HIV were positive for precancerous cervical cancer [8].

Despite its preventable cause, global cervical cancer incidence rate is expected to be doubled by 2025 [12]. While performing regular screening is known to prevent the disease by a significant percentage $[13,14]$, the acceptability of regular screening in Ethiopia is limited and covers less than $1 \%$ of women $[12,15]$. Moreover, comprehensive data on knowledge of women living with HIV/AIDS regarding cervical cancer and acceptability of screening is lacking in Ethiopia, which limits the development of cancer prevention efforts in these patient populations. Hence, the present study was aimed at assessing the level of knowledge about cervical cancer and uptake of screening among women living with HIV/AIDS in Gondar, northwest Ethiopia.

\section{Methods}

\section{Study design and setting}

A hospital-based cross-sectional survey was employed on 302 women attending ART clinic at University of Gondar Referral and Teaching Hospital (UoGRTH), northwest Ethiopia. UoGRTH is located in Gondar town, northwest Ethiopia, $738 \mathrm{~km}$ away from Addis Ababa (the capital city of Ethiopia).

\section{Sampling and recruitment strategies}

All HIV infected women above age of 17 years who visited the outpatient clinic of UoGRTH for follow up and medication refill were taken as a study population. Single population proportion formula was used with the assumption of $95 \%$ confidence interval, $5 \%$ margin of error, the proportion (p) of cervical cancer screening in women living with HIV/AIDS (11\%) [16] and 5\% for possible nonresponse was taken to determine a final sample size of 317. A systematic random sampling technique was then applied to select participants until the final sample size was attained.

\section{Survey instrument}

Data collection was performed by two of the principal investigators through interviewer-administered questionnaire. The investigators were properly trained on the instrument and ways of approaching the patients and securing potential participants permission for the interview prior to the commencement of the study. The data collection tool was developed after a thorough literature review of the published studies [17-20] and was primarily prepared in English. This was translated to local language (Amharic) and then back to English by an expert in the area in order to ensure that the translated version gives the proper meaning. The data collection instrument was also pretested on 20 women who were not included in the final analysis and relevant modifications were instituted before the commencement of actual data collection. The final questionnaire divided into three main parts. The first section was focusing on the socio-demographic and disease related information including age, marital status, educational level, CD4 count and WHO clinical stage. The second section, having 30 yes/no or true/false questions, assessed the knowledge about cervical cancer (CC) screening with five subcategories (risk factors for $\mathrm{CC}$, prevention of $\mathrm{CC}$, clinical symptoms of $\mathrm{CC}$, benefits of screening, and meaning of positive results). The third section asked respondents regarding the uptake of cervical cancer screening services. Total scores for each category were then summed up to determine an overall score with a maximum score of 30 . Using published literature as a reference [17], we classify respondents with a score of $60 \%$ or more as knowledgeable. The number of women who achieved this for any one score is defined as the knowledge rate. The third section included questions about the respondents' uptake of $\mathrm{CC}$ screening.

\section{Statistical analysis}

All the statistical analyses were done using Statistical Package for the Social Sciences (SPSS) software version 21.0 for Windows (SPSS Inc., Chicago, IL). Frequencies and percentages were used to express different variables. Univariate and multivariate logistic regression analysis were used to determine predictors of knowledge about cervical cancer and acceptability of screening. The results were adjusted for patients' demographic and 
disease characteristics. Odds ratio (OR) with 95\% CI were computed along with corresponding $p$-value $(p<$ $0.05)$ as cut off points for determining statistical significance.

\section{Ethical considerations}

This study was approved by the ethical review committee of University of Gondar with an approval number of UoGSoP-92/2017. Written informed consent from the respondents was also obtained before conducting this study. Participants' information obtained was kept confidential.

\section{Operational definitions}

CD4 Count: It is a laboratory value that measures the number of CD4 T lymphocytes (CD4 cells) in a sample of blood. In people with $\mathrm{HIV}$, the CD4 count is the most important laboratory indicator of immune function and the predictor of HIV progression. Healthy individuals CD4 count ranges from 500 to 1700 cells $/ \mathrm{mm}^{3}$. However, in HIV positive individuals the CD4 count sharply drops down to less than 500 cells $/ \mathrm{mm}^{3}$, which shows immunodefiency.

WHO clinical Stage: WHO clinical staging is based on clinical findings that guide the diagnosis, evaluation, and management of HIV/AIDS, and it does not require a CD4 cell count. This staging system is used in many countries to determine eligibility for antiretroviral therapy, particularly in settings in which CD4 testing is not available like the case of Ethiopia. These stages are defined by specific clinical conditions or symptoms. With this, clinical stages are categorized as 1 through 4, progressing from primary HIV infection to advanced HIV/AIDS [21].

\section{Results}

\section{Characteristics of the study participants}

Out of 317 patients approached, 302 of them were included in the study giving a response rate of $95.3 \%$. The mean age of respondents was 33.72 years with a standard deviation of \pm 9.72 . Majority of the respondents were urban residents (69.9\%). A substantial proportion of respondents $(36.7 \%)$ were at the stage of WHO clinical stage 2 and had a CD4 count of $\leq 500$ cells/ul (66.9\%). The sociodemographic and disease characteristics of study participants are depicted in Table 1.

\section{Knowledge and uptake of cervical cancer screening}

The majority of respondents in this study 265 (87.7\%) had heard about cervical cancer and its screening. The average total knowledge score was found to be $10.8 \pm 5.20$ (a range possible from 0 to 30 ), with a mean score of $1.64 \pm 1.27$ for the risk factors of $\mathrm{CC}$ (a range possible from 0 to 7 ), $1.77 \pm 1.81$ for preventive measures of CC (a range possible from 0 to 7 ), $2.74 \pm 1.67$ for clinical symptoms of $\mathrm{CC}$ (a range possible from 0 to 9), $1.71 \pm 1.23$ for benefits of screening (a range possible from 0 to 3 ) and $2.72 \pm 1.07$
Table 1 Sociodemographic characteristics and factors associated with uptake of cervical cancer screening, Gondar, 2017

\begin{tabular}{|c|c|c|c|c|}
\hline \multirow[t]{2}{*}{ Variables } & \multirow[t]{2}{*}{ Total } & \multicolumn{2}{|l|}{ Screening } & \multirow[t]{2}{*}{ AOR $(95 \% \mathrm{Cl})$} \\
\hline & & $\begin{array}{l}\text { No } \\
(n=231)\end{array}$ & $\begin{array}{l}\text { Yes } \\
(n=71)\end{array}$ & \\
\hline \multicolumn{5}{|l|}{ Age group, in years } \\
\hline$<29$ & $128(42.4 \%)$ & 111 & 17 & 1 \\
\hline $30-39$ & $105(34.8 \%)$ & 69 & 36 & $2.78(1.71-7.29)$ \\
\hline$>40$ & $69(22.8 \%)$ & 51 & 18 & $2.61(1.89-5.17)$ \\
\hline \multicolumn{5}{|l|}{ Residence } \\
\hline Rural & $91(30.1 \%)$ & 71 & 20 & - \\
\hline Urban & $211(69.9 \%)$ & 160 & 51 & - \\
\hline \multicolumn{5}{|l|}{ Marital status } \\
\hline Unmarried & $123(40.7 \%)$ & 112 & 11 & - \\
\hline Ever married & $179(59.3 \%)$ & 119 & 60 & - \\
\hline \multicolumn{5}{|l|}{ Educational status } \\
\hline Illiterate & $33(10.9 \%)$ & 28 & 5 & 1 \\
\hline Primary & $145(48 \%)$ & 133 & 12 & $0.87(0.33-1.79)$ \\
\hline Secondary & $80(26.5 \%)$ & 66 & 14 & $1.08(0.54-1.91)$ \\
\hline Tertiary & $44(14.6 \%)$ & 4 & 40 & $0.41(0.21-1.29)$ \\
\hline \multicolumn{5}{|c|}{ Average monthly income } \\
\hline$<100$ & $148(49 \%)$ & 119 & 29 & - \\
\hline $100-150$ & $85(28.2 \%)$ & 59 & 16 & - \\
\hline$>150$ & $69(22.8 \%)$ & 43 & 26 & - \\
\hline \multicolumn{5}{|l|}{ Age at first sex } \\
\hline$\leq 16$ & $63(20.9 \%)$ & 46 & 17 & - \\
\hline$>16$ & $239(79.1 \%)$ & 185 & 54 & - \\
\hline \multicolumn{5}{|c|}{ Had multiple sexual partner } \\
\hline No & $123(40.7 \%)$ & 89 & 34 & 1 \\
\hline Yes & $179(59.3 \%)$ & 142 & 37 & $1.01(0.43-1.72)$ \\
\hline \multicolumn{5}{|l|}{$\begin{array}{l}\text { Comprehensive } \\
\text { knowledge about CC }\end{array}$} \\
\hline Not knowledgeable & $238(78.8 \%)$ & 129 & 9 & 1 \\
\hline Knowledgeable & $64(21.2 \%)$ & 2 & 62 & $3.02(2.31-7.15)$ \\
\hline \multicolumn{5}{|l|}{ CD4 count } \\
\hline$<500$ cells/ul & $202(66.9 \%)$ & 170 & 32 & - \\
\hline$>500$ cells/ul & $100(33.1 \%)$ & 61 & 39 & - \\
\hline \multicolumn{5}{|l|}{ WHO clinical stage } \\
\hline One & 91 (30.1\%) & 80 & 11 & 1 \\
\hline Two & $111(36.7 \%)$ & 90 & 21 & $0.62(0.39-1.72)$ \\
\hline Three & $67(22.2 \%)$ & 52 & 15 & $1.01(0.41-1.52)$ \\
\hline Four & $33(11 \%)$ & 9 & 24 & $0.91(0.40-1.69)$ \\
\hline \multicolumn{5}{|l|}{ Perceived susceptibility } \\
\hline None receptive & $118(39.1 \%)$ & 105 & 13 & 1 \\
\hline Receptive & 184 (60.9\%) & 126 & 58 & $2.85(1.89-6.16)$ \\
\hline
\end{tabular}

Comprehensive

nowledge about CC 
for understanding about the positive results of CC (a range possible from 0 to 4 ). Overall, only 64 (21.2\%) of respondent were knowledgeable about CC and screening as per the definition set in our study.

The majority of respondents correctly answered that CC is both preventable 238 (78.8\%) and curable 223 (73.8\%) disease. However, a significant proportion of respondents 103 (34.1\%) didn't know the risk factors of CC or identified only one $57(18.9 \%)$ or two $45(14.9 \%)$ risk factors. While a highest proportion of patients correctly identified "early onset of sexual activity" 112 (37.1\%) as a risk factor for CC, hormonal contraceptive use and HPV infection were identified as risk factors for CC only by $57(18.9 \%)$ and $53(17.5 \%)$ of respondents respectively. Similarly, over half of the respondents 172 (56.9\%) knew that CC screening could prevent CC occurrence. Yet, only 88 (29.1\%) respondents believed that $\mathrm{CC}$ screening could enable early diagnosis of the disease. Even though majority of respondents knew at least one of the clinical symptoms of CC $274(90.7 \%)$, a substantial proportion of respondents 63 (20.8\%) incorrectly stated "vulvar itching or burning sensation" as one of the clinical symptoms of CC. The detailed frequency of correct answer for each knowledge items are presented in Table 2. According to the findings our study, only $71(23.5 \%)$ of respondents were ever tested for CC in their life time, of which $29(40.8 \%)$ of them screened after 1 year of HIV/AIDS diagnosis. Among the 231 (76.5\%) of respondents who were not screened for CC, absence of symptoms 205 (88.7\%) and emotional barriers like fear of test result 164 (71\%) and embarrassment 159 (68.8\%) were the main reasons for not undergoing CC screening (Table 3).

\section{Predictors of CC screening service uptake}

Logistic regression analysis was employed to assess possible associations between different sociodemographic variables and women's CC screening service uptake. According to the results from bivariate logistic regression, there were statistically significant differences in age, history of multiple sexual partners, educational status, WHO clinical stage, comprehensive knowledge of $\mathrm{CC}$ and screening and perceived susceptibility to develop $\mathrm{CC}$ between women who underwent $\mathrm{CC}$ screening and those who didn't. Variables that were significantly associated with $\mathrm{CC}$ screening service uptake in the bivariate analysis (those with $p$-value $<0.20$ ) were further examined in multivariate logistic regression. Accordingly, age, perceived susceptibility to develop CC and comprehensive knowledge of $\mathrm{CC}$ and screening remained to be significant in the multivariate logistic model. The odds of CC screening service uptake among women in the age range of 30-39 years were 1.78 times higher than women aged less than 29 years old ( $\mathrm{AOR}=2.78,95 \% \mathrm{CI}$ $=1.71-7.29$ ). The odds of CC screening uptake among
Table 2 Frequency of correct answer for knowledge items about CC among participants, Gondar, Ethiopia, 2017

\begin{tabular}{|c|c|}
\hline Knowledge items & $\begin{array}{l}\text { Correct } \\
\text { answers (\%) }\end{array}$ \\
\hline \multicolumn{2}{|l|}{ Risk factor for CC } \\
\hline Prolonged use of oral contraceptive & $57(18.9 \%)$ \\
\hline Sexually transmitted infection & $77(25.5 \%)$ \\
\hline Early onset of sexual activity & $112(37.1 \%)$ \\
\hline Smoking & $54(17.9 \%)$ \\
\hline Multiple sexual partner & 49 (16.2\%) \\
\hline History of HPV infection & $53(17.5 \%)$ \\
\hline Aged 30-65 & $65(21.5 \%)$ \\
\hline \multicolumn{2}{|l|}{ Symptoms of cervical cancer } \\
\hline Bleeding and pain after sexual intercourse & $60(19.9 \%)$ \\
\hline Vulvar itching or burning sensation & $63(20.8 \%)$ \\
\hline Post-menopausal bleeding & $54(17.9 \%)$ \\
\hline Excessive vaginal discharge & $71(23.5 \%)$ \\
\hline Abnormal vaginal discharge & $68(22.5 \%)$ \\
\hline Inter-menstrual bleeding & $67(22.2 \%)$ \\
\hline Longer or heavier menstrual periods & $55(18.2 \%)$ \\
\hline Pelvic pain & $48(15.9 \%)$ \\
\hline Urinary frequency, urgency & $38(12.6 \%)$ \\
\hline \multicolumn{2}{|l|}{ Preventive measures for CC } \\
\hline CC screening & $172(56.9 \%)$ \\
\hline Reduce numbers of sexual partners & $61(20.2 \%)$ \\
\hline Vaccine for HPV & $27(8.9 \%)$ \\
\hline Late marriage and late childbirth & $21(6.9 \%)$ \\
\hline No smoking & $57(18.9 \%)$ \\
\hline Consistent condom use & $29(9.6 \%)$ \\
\hline Prompt treatment of STIs & $71(23.5 \%)$ \\
\hline \multicolumn{2}{|l|}{ Benefits of screening for CC } \\
\hline Early detection & $71(23.5 \%)$ \\
\hline Early diagnosis & $88(29.1 \%)$ \\
\hline Early treatment & $101(33.4 \%)$ \\
\hline \multicolumn{2}{|l|}{ Understanding of the positive results } \\
\hline $\begin{array}{l}\text { Negative screening result means cervix without any } \\
\text { lesion, needing no more screening }\end{array}$ & $142(47 \%)$ \\
\hline Positive screening result means suffering from CC & $224(74.2 \%)$ \\
\hline $\begin{array}{l}\text { Positive screening result means there is cervical lesion, } \\
\text { it needs further diagnosis }\end{array}$ & $76(25.2 \%)$ \\
\hline$C C$ is a curable disease & $223(73.8 \%)$ \\
\hline
\end{tabular}

women who had positive perception on their susceptibility to develop CC were 1.85 times higher than those who had negative perception $(\mathrm{AOR}=2.85,95 \% \mathrm{CI}=$ 1.89-6.16). Similarly, the odds of undergoing CC screening among women who had a comprehensive knowledge on CC and screening were 2.02 times higher than those 
Table 3 Acceptance of CC screening service among study participants, Gondar, Ethiopia, 2017

\begin{tabular}{lc}
\hline Variables & Frequency (\%) \\
\hline Have you ever had CC screening in your life time? \\
No & $231(76.5 \%)$ \\
Yes & $71(23.5 \%)$ \\
If yes, when was the last time you screened for cervical cancer? (N=71) \\
Before HIV/AIDS diagnosis & $19(26.8 \%)$ \\
Within 1 year of HIV/AIDS diagnosis & $23(32.4 \%)$ \\
After 1 year of HIV/AIDS diagnosis & $29(40.8 \%)$ \\
If no, what are the reasons for not being screened? (N=231) \\
Absence of symptoms & $205(88.7 \%)$ \\
High cost of the test & $64(27.7 \%)$ \\
Not prescribed by the doctor & $76(32.9 \%)$ \\
Embarrassment & $159(68.8 \%)$ \\
Time consuming & $44(19 \%)$ \\
Fear of test result & $164(71 \%)$ \\
Screening center too far & $87(37.7 \%)$ \\
No reason & $46(19.9 \%)$ \\
Others & \\
Are you willing to be screened in the near future? & $1998.2 \%)$ \\
No & $88(29 \%)$ \\
Yes & $214(71 \%)$ \\
\hline
\end{tabular}

${ }^{\mathrm{a} O t h e r s ~ i n c l u d e ~ R e l i g i o u s ~ d e n i a l, ~ p a r t n e r ~ a c c e p t a n c e, ~ n o ~ s y m p t o m ~}$

who didn't have comprehensive knowledge on $\mathrm{CC}$ and screening $(\mathrm{AOR}=3.02,95 \% \mathrm{CI}=2.31-7.15)$.

\section{Discussion}

Exploring the comprehensive knowledge towards the causative/risk factors, benefits of screening, pertinent manifestations and prevention of cervical cancer is so indispensable in women care. According to the finding of this study, majority of $(87.7 \%)$ respondents heard about cervical cancer and its screening. The overall knowledge rate of CC was $21.2 \%$, which is higher compared to the study done in Nigeria where $11.8 \%$ of the rural and $17.6 \%$ of urban women had knowledge of CC [19]. In line with this, the average total knowledge score women had was found to be merely $10.8 \pm 5$, which is higher than the study conducted in china, which is $6.91 \pm 3.42$ [18]. The difference in knowledge score could be attributed to the difference in time period and the characteristics of the study population as both of the studies were conducted among the general population in Nigeria and China and did not account whether the respondents had HIV/AIDS or not. This could potentially account for the variation in the level of knowledge as patients living with HIV/AIDS expected to have a higher level of awareness about CC due to their frequent contact with healthcare providers compared to the general population. In our study, only
$33.8 \%$ of the study respondents were capable of identifying one or two risk factors for $\mathrm{CC}$, which is lower than the study conducted among rural communities of South Africa in which about $64 \%$ of the respondents gave one or more risk factors [22]. This might be due to the absence of a comprehensive cancer prevention and treatment center in Ethiopia unlike countries like South Africa..Majority of respondents in this study (73.8\%) believed that CC is curable, which is lower than the study conducted in China $(80.8 \%)$ [18]. This might be due to the difference in the background of study population as the study conducted in China included respondents from Wufeng, a high-incidence region of cervical cancer in China.

According to the findings of our study, only $23.5 \%$ of respondents were ever tested for CC in their life time, which is significantly higher compared with the study done in among patients living with HIV/AIDS in Addis Ababa, Ethiopia, where only $11.5 \%$ of women screened for CC [16]. The uptake of screening in our study is also higher compared with the study conducted in Nigeria (9.4\%) [23]. The enhanced uptake of screening service in our study could be partially explained by the increased nation-wide advocacy, community sensitization and awareness creation about the $\mathrm{CC}$ screening that has been put into effect in recent years. It might also be due to the improved expansion and access of screening centers across the country and integration of $\mathrm{CC}$ screening into the standard care for women who are living with HIV/AIDS. Yet, the proportion of women screened for CC in our study is still low compared to developed countries such as Ottawa (58\%) [24], despite the recent effort to screen all HIV positive women who are on antiretroviral therapy (ART) who were not screened before. Among $76.5 \%$ of patients who were not screened in our study, absence of symptoms (88.7\%) and emotional barriers like fear of test result (71\%) and embarrassment (68.8\%) were the main reasons for not undergoing screening, which was consistent with the study conducted in China [18]. According to the results from multivariate logistic regression analysis, age, perceived susceptibility to develop CC and comprehensive knowledge of $\mathrm{CC}$ and screening remained to be strong predictors of $\mathrm{CC}$ screening service uptake. The odds of CC screening service uptake among women in the age range of 30-39 years were 2.78 times higher than women aged 21-29 years old. Similar findings were also reported both in developing and developed countries [25, 26]. This is not surprising as women at the age of $30 \mathrm{~s}$ and $60 \mathrm{~s}$ are more likely to be symptomatic due to the bimodal distribution nature of the $\mathrm{CC}$, which may enhance their probability of screening for CC. Similarly, the odds of CC screening uptake among women who had positive perception on their susceptibility to develop CC were 2.85 times higher than those who had negative perception, which could be explained by the assumption of behavioral model, which assumes that belief and attitudes, including self-vulnerability 
to illness, are important predictors of their health-related activities [27]. Furthermore, the odds of undergoing CC screening among women who had a comprehensive knowledge on $\mathrm{CC}$ and screening were 3.02 times higher than those who didn't have comprehensive knowledge on CC and screening, which corroborates the findings of studies conducted among patients living with HIV/AIDS in Addis Ababa, Ethiopia and Botswana $[16,28]$.

\section{Limitation of the study}

Even though this survey highlights an area of research where there is lack of literature in Ethiopia, caution should be exercised when generalizing to other regions in Ethiopia as the study was a cross-sectional and conducted only in Gondar, northwest Ethiopia. Nevertheless, this survey has significant implications for improving uptake of CC screening services and provide a foundation for planning future in-depth research prior to developing educational materials. A larger-scale and multi centered survey that includes more diverse participants is warranted to validate our findings and to provide more accurate findings. Furthermore, our study could be used as an input for future studies aiming at exploring the difference in knowledge about cervical cancer and cervical cancer screening uptake experience between HIV positive and negative women.

\section{Conclusion and recommendation}

The results of the present study revealed that the knowledge and uptake of cervical cancer screening among HIV infected women was very poor. Our findings emphasize the need to reform the existing national strategies of cervical cancer screening so as to strengthen the health education and promotion, beyond providing screening services. Taking into consideration the heightened importance of comprehensive knowledge in participating in cervical cancer screening services, different stakeholders working on cancer and HIV/AIDS should provide a customized health promotion intervention and awareness creation among HIV-infected women. Furthermore, interventions should focus on overcoming the identified barriers for not being screened including improving accessibility of cervical cancer screening services in rural areas.

\section{Abbreviations \\ CC: Cervical cancer; Cl: Confidence interval; HIV/AIDS: Human immunodeficiency virus/Acquired immune deficiency syndrome; HPV: Human papillomavirus; HR-HPV: High risk oncogenic Human papillomavirus; OR: Odds ratio; SPSS: Statistical package for the social sciences; UoGRTH: University of Gondar Referral and Teaching Hospital; WHO: World health organization}

\section{Acknowledgements}

The authors acknowledge the Support of School of pharmacy, University of Gondar and UOGRTH in facilitating the data collection process.

Funding

No financial support was gained to conduct this study.
Availability of data and materials

All relevant materials and data supporting the findings of this study are contained within the manuscript.

\section{Authors' contributions}

DAE, SAB involved in conceptualization, project administration, formal analysis, investigation, methodology and supervision; ABM, SAA and AKN involved in data curation, resources, writing and original draft of the manuscript; $A G M$ and DAE involved in methodology, investigation and writing, review \& editing of the final manuscript. All authors read and approved the final manuscript.

Ethics approval and consent to participate

This study was approved by the ethical committee of University of Gondar. Written informed consent from the patients was also obtained before conducting this study. Participants' information obtained was kept anonymous.

\section{Consent for publication}

Not applicable.

\section{Competing interests}

The authors declare that they have no competing interests.

\section{Publisher's Note}

Springer Nature remains neutral with regard to jurisdictional claims in published maps and institutional affiliations.

\section{Author details}

'Department of Clinical Pharmacy, School of Pharmacy, College of Medicine and Health Sciences, University of Gondar, Chechela Street, Lideta Sub city Kebele 16, P.O. Box: 196, Gondar, Ethiopia. ²Department of Gynecology and obstetrics, College of Medicine and Health Sciences, University of Gondar, Chechela Street, Lideta Sub city Kebele 16, Gondar, Ethiopia. ${ }^{3}$ Department of Pharmacology, School of Pharmacy, University of Gondar, Chechela Street, Lideta Sub city Kebele 16, Gondar, Ethiopia.

Received: 21 July 2017 Accepted: 4 December 2017

Published online: 19 December 2017

\section{References}

1. Integrated Africa Cancer Factsheet. Focusing on cervical cancer, Girls \& Women Health, Sexual \& Reproductive Health, HIV \& Maternal Health. 2014.

2. WHO. International agency for research on cancer: Latest world cancer statistics; 2013.

3. Zur Hausen H. Papillomaviruses in the causation of human cancers - a brief historical account. Virology. 2009;384:260-5.

4. Bruni L, Barrionuevo-Rosas L, Albero G, Aldea M, Serrano B, Valencia S, et al. ICO information Centre on HPV and cancer (HPV information Centre). Human Papillomavirus and Related Diseases in Ethiopia. 2014;12:18. Summary Report http://www.hpvcentre.net/.

5. Tanon A, Jaquet A, Ekouevi DK, Akakpo J, Adoubi I, et al. The pectrum of cancers in West Africa: associations with human immunodeficiency virus. PLoS One. 2012;7(10):e48108.

6. Moscicki AB, Ellenberg JH, Vermund SH, Holland CA, Darragh T, CrowleyNowick PA, et al. Prevalence of and risks for cervical human papillomavirus infection and squamous intraepithelial lesions in adolescent girls: impact of infection with human immunodeficiency virus. Arch Pediatr Adolesc Med. 2000;154(2):127-34.

7. Meijer CJ, Rozendaal L, Voorhorst FJ, Verheijen R, Helmerhorst TJ, Walboomers JM. Human papillomavirus and screening for cervical cancer: state of art and prospects. Ned Tijdschr Geneeskd. 2000;144(35):1675-9.

8. Gedefaw A, Astatkie A, Tessema GA. The prevalence of precancerous cervical cancer lesion among HIV-infected women in southern Ethiopia: a cross-sectional study. PLoS One. 2013;8(12):e84519.

9. Sun X-W, Kuhn L, Ellerbrock TV, Chiasson MA, Bush TJ, Wright TC Jr. Human Papillomavirus infection in women infected with the human immunodeficiency virus. N Engl J Med. 1997;337:1343-9.

10. Sam MM, Kishor B, Clement A, Annie JS. HIV and cancer in Africa: mutual collaboration between HIV and cancer programs may provide timely research and public health data. Infect Agents Cancer. 2011;6:16. https://doi. org/10.1186/1750-9378-6-16. 
11. UNAIDS. AIDS epidemic update Geneva, Switzerland. November 2009. http://www.unaids.org/. Global report UNAIDS report on the global aids epidemic. 2010. http://www.unaids.org/en.

12. WHO/ICO Information Centre on HPV and Cervical Cancer (HPV Information Centre). Human Papillomavirus and Related Cancers in World. Summary Report 2010. http://www.hpvcentre.net.

13. Cancer Research UK Registered charity in England and Wales (1089464), Scotland (SC041666) and the Isle of Man (1103). 2014. cruk.org/cancerstats.

14. Minjee L, Eun-Cheol P, Hoo-Sun C, Jeoung AK, Ki Bong Y, et al. Socioeconomic disparity in cervical cancer screening among Korean women: 1998-2010. MC Public Health. 2013;13:553.

15. Joint United Nations Program on HIV/AIDS. (UNAIDS) report on the global AIDS epidemic. 2010.

16. Belete $\mathrm{N}$, Tsige $\mathrm{Y}$, Mellie H. Willingness and acceptability of cervical cancer screening among women living with HIV/AIDS in Addis Ababa, Ethiopia: a cross sectional study. Gynecologic Oncology Research and Practice. 2015;2: 6. https://doi.org/10.1186/s40661-015-0012-3.

17. Di J, Rutherford S, Wu J, Song B, Ma L, Chen J, et al. Knowledge of cervical cancer screening among women across different socio- economic regions of China. PLoS One. 2015;10(12):e0144819. https://doi.org/10.1371/journal. pone.0144819.

18. Jia Y, Li S, Yang R, Zhou H, Xiang QY, Hu T, et al. Knowledge about cervical cancer and barriers of screening program among women in Wufeng County, a high-incidence region of cervical cancer in China. PLoS One. 2013;8(7):e67005. https://doi.org/10.1371/journal.pone.0067005.

19. Nwankwo K, Aniebue U, Aguwa E, Anarado A, Agunwah E. Knowledge attitudes and practices of cervical cancer screening among urban and rural Nigerian women: a call for education and mass screening. European journal of cancer care. 2011;20(3):362-7. https://doi.org/10.1111/j.1365-2354.2009. 01175.x.

20. Getahun F, et al. Comprehensive knowledge about cervical cancer is low among women in Northwest Ethiopia. BMC Cancer. 2013;13:2.

21. HIV Classification: CDC and WHO Staging Systems https://www.aidsetc.org/ guide/hiv-classification-cdc-and-who-staging-systems.

22. Hoque $M$, Hoque E, Kader SB. Evaluation of cervical cancer screening program at a rural community of South Africa. East Afr J Publ Health. 2008;5(2):111.

23. Oliver CE, Chidinma VGO, Per OO, Karen OP. Willingness and acceptability of cervical cancer screening among HIV positive Nigerian women. BMC Public Health. 2013;13:46.

24. Pamela L, Claire K, Claire T, Kevin P, Jonathan BA, James J. Cervical cancer screening among HIV-positive women retrospective cohort study from a tertiary care HIV clinic. Can Fam Physician. 2010;56:e425-31.

25. Sawaya GF, Sung H-Y, Kathleen A, Marie M, Walter K, Robert A, et al. Advancing age and cervical cancer screening and prognosis. J Am Geriatr Soc. 2001:49:1499-504.

26. Smith AM, Heywood W, Ryall R, Shelley JM, Pitts MK, Richters J, et al. Association between sexual behavior and cervical cancer screening. J Women's Health. 2011;20:1091-6.

27. Ndikom CM, Ofi BA. Awareness, perception and factors affecting utilization of cervical cancer screening services among women in Ibadan, Nigeria: a qualitative study. Reprod Health. 2012;9:11.

28. Mingo AM, Panozzo CA, Taylor DiAngi Y, Smith J, Steenhoff AP, et al. Cervical cancer awareness and screening in Botswana. International journal of gynecological cancer: official journal of the International Gynecological Cancer Society. 2012;22:638-51.

\section{Submit your next manuscript to BioMed Central and we will help you at every step:}

- We accept pre-submission inquiries

- Our selector tool helps you to find the most relevant journal

- We provide round the clock customer support

- Convenient online submission

- Thorough peer review

- Inclusion in PubMed and all major indexing services

- Maximum visibility for your research

Submit your manuscript at www.biomedcentral.com/submit
Biomed Central 